\title{
Factores asociados a la obesidad en perros adultos de Lima, Perú
}

\author{
Factors associated with obesity in adult dogs from Lima, Peru
}

\author{
Lia Segami C..$^{1,3}$, Roberto Dávila F. ${ }^{1}$, Boris Lira M. ${ }^{2}$
}

\section{Resumen}

El objetivo del estudio fue determinar los factores asociados a la presentación de obesidad en perros atendidos en la Clínica de Animales Menores de la Facultad de Medicina Veterinaria de la Universidad Nacional Mayor de San Marcos durante 2017. Se trabajó con 89 perros y se determinó el sexo, raza y condición corporal; asimismo, la edad, actividad física, esterilización (sí/no) y tipo de alimentación fueron obtenidos mediante una encuesta a los dueños de los perros. La condición corporal estimada permitió clasificar a los perros entre obesos y no obesos. La edad promedio fue 7.7 años ( 0 -16 años), siendo $50.6 \%(n=45)$ de sexo hembra. El 47.2\% $(n=42)$ estaba esterilizado y $28.1 \%(n=25)$ fue identificado como mestizo. El 46.1\% ( $=41)$ consumía alimento balanceado como parte de su dieta y el $74.2 \%(\mathrm{n}=66)$ realizaba actividad física de manera regular. El $60.7 \%$ $(n=54)$ fue considerado obeso. Se evidenciaron asociaciones significativas con la presentación de obesidad para una dieta basada en alimento casero [OR 11.5; 95\%IC: 2.9644.65], raza pura en comparación a los mestizos [OR 4.34; 95\% CI: 1.03-18.32] y edad entre 8 y 12 años en comparación con perros de 1 año [OR 20.13; 95\%CI:2.30-176.02]. No se encontró asociación con el sexo, actividad física y esterilización.

Palabras clave: obesidad, factores asociados, perros

${ }^{1}$ Clínica de Animales Menores, Facultad de Medicina Veterinaria, Universidad Nacional Mayor de San Marcos, Lima, Perú

${ }^{2}$ Laboratorio de Fisiología Animal, Facultad de Medicina Veterinaria, Universidad Nacional Mayor de San Marcos, Lima, Perú

${ }^{3}$ E-mail: segami.lia@gmail.com; https://orcid.org/0000-0002-0929-0636

Recibido: 22 de septiembre de 2020

Aceptado para publicación: 26 de febrero de 2021

Publicado: 23 de junio de 2021

CLos autores. Este artículo es publicado por la Rev Inv Vet Perú de la Facultad de Medicina Veterinaria, Universidad Nacional Mayor de San Marcos. Este es un artículo de acceso abierto, distribuido bajo los términos de la licencia Creative Commons Atribución 4.0 Internacional (CC BY 4.0) [https:// creativecommons.org/licenses/by/4.0/deed.es] que permite el uso, distribución y reproducción en cualquier medio, siempre que la obra original sea debidamente citada de su fuente original 
The aim of this study was to determine the factors associated with the presentation of obesity in dogs treated at the Small Animal Clinic of the Faculty of Veterinary Medicine of the Universidad Nacional Mayor de San Marcos during 2017. In total, 89 dogs were included in the study. Sex, breed and body condition was recorded. Likewise, age, physical activity, neutering (yes/no) and type of diet were obtained through a survey applied to dog owners. The estimated body condition allowed the dogs to be classified between obese and non-obese. The average age was 7.7 years $(0-16$ years $)$, with $50.6 \%(n=45)$ being female. Besides, $47.2 \%(n=42)$ were neutered and $28.1 \%(n=25)$ were crossbreds. Results showed that $46.1 \%(n=41)$ consumed balanced food as part of their diet and $74.2 \%(n=66)$ carried out regular physical activity. A total of $60.7 \%(n=54)$ were considered obese. Significant associations with the presentation of obesity were evidenced for a diet based on homemade food [OR 11.5; 95\% CI: 2.96-44.65], purebreds compared to crossbreds [OR 4.34; 95\% CI: 1.03-18.32] and age between 8 and 12 years compared to 1year-old dogs [OR 20.13; 95\% CI: 2.30-176.02]. No association was found with sex, physical activity and neutering.

Key words: obesity, associated factors, dogs

\section{INTRODUCCIÓN}

La obesidad se define como la acumulación de cantidades excesivas de tejido adiposo en el cuerpo y es el desorden nutricional más común en animales de compañía (Gossellin et al., 2007; Zoran 2010). Es usualmente el resultado de una ingesta alimentaria excesiva, generando un desequilibrio energético positivo (German, 2001). Similar a humanos, la obesidad en perros trae consigo detrimento en la salud con efectos en enfermedades asociadas como osteoartritis, diabetes mellitus y algunos tipos de neoplasia (Kopelman, 2000; German et al., 2012). Adicionalmente, la frecuencia de perros con sobrepeso y obesidad ha aumentado en los últimos años (Corbee, 2012; Wakshlag y Loftus, 2014).

En medicina veterinaria, el sobrepeso y obesidad en perros son problemas que frecuentemente pasan desapercibidos en la práctica diaria (German et al., 2018). No es común recabar información relacionada a la condición corporal, y rara vez es registrado en las fichas clínicas (Rolph et al., 2014). Adicionalmente, los dueños no perciben adecuadamente el peso de sus mascotas, siendo esto más común en perros con sobrepeso (Eastland-Jones et al., 2014).

Las hembras son más propensas que los machos a la obesidad (Mao et al., 2013; Bjørnvad et al., 2019), en tanto que la esterilización (Mao et al., 2013; Bjørnvad et al., 2019) y la edad avanzada (Courcier et al., 2010; Mao et al., 2013) han sido identificados como factores de riesgo adicionales. Según el tipo de alimento, se menciona que el consumo de alimento casero predispone a la obesidad (Lund et al., 2006; Sallander et al., 2010), aunque hay estudios que no han encontrado diferencias entre el uso de alimento casero y comercial como causal de obesidad (Gerstner y Liesegang, 2017). La actividad física, de otra parte, se ha visto asociada con una disminución de riesgo de obesidad (Mao et al., 2013; Raffan et al., 2016), aunque esto no pudo ser confirmado en otros estudios (Sallander et al., 2010; Gerstner y Liesegang, 
2017). Algunas razas han sido asociadas con el desarrollo de obesidad, aunque con grandes variaciones entre países (Reiter et al., 2016); así en el Perú existe una alta proporción de perros mestizos, condición que no es no incluida en muchos estudios.

Determinar los factores asociados brinda un mejor entendimiento de la epidemiología de la obesidad y ayuda a prevenir su presentación en poblaciones caninas (Lund et al., 2006). Por ello, el objetivo del presente estudio fue explorar los posibles factores asociados a la presentación de obesidad en perros atendidos en la Clínica de Animales Menores de la Facultad de Medicina Veterinaria de la Universidad Nacional Mayor de San Marcos.

\section{MATERIALES Y MéTOdOS}

El estudio se desarrolló en las instalaciones de la Clínica de Animales Menores (CAM) de la Facultad de Medicina Veterinaria de la Universidad Nacional Mayor de San Marcos (FMV-UNMSM) en Lima, Perú.

Se trabajó con 89 perros de uno a más años, atendidos en la CAM en 2017, así como con sus dueños, los cuales aceptaron participar de manera voluntaria en el estudio. Se registró el sexo, raza o tipo racial, y condición corporal del animal. Además se utilizó una encuesta para registrar la edad, actividad física, esterilización (sí/no) y tipo de alimentación. La condición corporal (CC) de los perros fue estimada en una escala de 9 puntos, siendo considerados como obesos cuando tuvieron una $\mathrm{CC}$ de 8 a 9 (Laflamme, 1997).

Toda la información recolectada fue digitalizada en una base de datos (Microsoft Excel). Para el análisis descriptivo, las variables categóricas fueron descritas mediante el uso de frecuencias. Se utilizó la prueba de Chi-cuadrado para examinar posibles asociaciones entre la obesidad y las otras variables incluidas en el estudio. Finalmente, se utilizó un modelo de regresión logística multivariado para el cálculo de Odds Ratio (OR) de obesidad para las variables con significancia estadística según el análisis previo o por asociación teórica conocida con la presentación de obesidad. Los OR estimados fueron considerados significativos si el intervalo de confianza (IC95\%) no incluyó el valor 1 (Szumilas, 2010). Los análisis fueron desarrollados utilizando el paquete estadístico $\mathrm{R}$ (v. 4.0.0) con un nivel de confianza de1 95\%.

\section{Resultados}

La edad promedio de los 89 perros fue 7.7 años (1-16 años). La edad fue distribuida en cinco categorías: menor o igual a 1 año $(\mathrm{n}=8,8.9 \%), 2$ a 3 años $(\mathrm{n}=13,14.6 \%), 4$ a 7 años $(n=21,23.6 \%), 8$ a 12 años $(n=36$, $40.5 \%)$ y mayores a 12 años $(\mathrm{n}=11,12.4 \%)$.

El 50.6\% ( $\mathrm{n}=45)$ fue hembra y, de ellas, el $60 \%(27 / 45)$ estaban esterilizadas. E1 $49.4 \%(n=44)$ fue macho y el $34.1 \%(15 / 44)$ se encontraba esterilizado. Las razas Cocker (16.9\%), Labrador Retriever (8.9\%), Schnauzer (8.9\%), Pequinés $(6.7 \%)$ y Shih Tzu (5.6\%) fueron las más representativas, en tanto que el $28.1 \%(n=25)$ fue considerado como mestizo.

El alimento casero fue el más empleado $(53.9 \% ; n=48)$, en tanto que los demás perros $(46.1 \%, \mathrm{n}=41)$ consumían alimento balanceado como parte de su dieta. De otra parte, el $74.2 \%(n=66)$ realizaba actividad física de manera regular, incluyendo caminatas y paseos.

Según la escala de Laflamme (1997), $60.7 \%(\mathrm{n}=54)$ de los perros fueron clasificados como obesos (CC 8-9) y $39.3 \%(n=35)$ con peso ideal (CC 4-5). El tipo de alimento se encontró asociado con la presentación de obesidad en caninos, siendo más frecuentes en dietas con alimento casero $68.5 \%(n=37)$ en comparación al $31.5 \%(\mathrm{n}=17)$ en perros que consumen alimento balanceado 
Cuadro 1. Análisis bivariado de factores asociados con la presentación de obesidad en perros adultos atendidos en la Clínica de Animales Menores de la Universidad Nacional Mayor de San Marcos (Lima, Perú, 2017)

\begin{tabular}{|c|c|c|c|}
\hline \multirow[t]{2}{*}{ Características } & \multicolumn{2}{|c|}{ Obesidad } & \multirow[t]{2}{*}{ valor $\mathrm{p}^{1}$} \\
\hline & No $(n=35)$ & Sí $(\mathrm{n}=54)$ & \\
\hline \multicolumn{4}{|l|}{ Sexo } \\
\hline Hembra & $17(48.6)$ & $28(51.9)$ & 0.830 \\
\hline Macho & $18(51.4)$ & $26(48.1)$ & \\
\hline \multicolumn{4}{|l|}{ Edad (años) } \\
\hline 1 & $6(17.1)$ & $2(3.7)$ & 0.030 \\
\hline $2-3$ & $6(17.1)$ & $7(12.9)$ & \\
\hline $4-7$ & $11(31.4)$ & $10(18.5)$ & \\
\hline $8-12$ & $8(22.9)$ & $28(51.9)$ & \\
\hline$>12$ & $4(11.4)$ & $7(12.9)$ & \\
\hline \multicolumn{4}{|l|}{ Esterilización } \\
\hline No & $22(62.9)$ & $25(46.3)$ & 0.136 \\
\hline Sí & $13(37.1)$ & $29(53.7)$ & \\
\hline \multicolumn{4}{|l|}{ Raza } \\
\hline Mestizo & $11(31.4)$ & $14(25.9)$ & 0.633 \\
\hline Pura & $24(68.6)$ & $40(74.1)$ & \\
\hline \multicolumn{4}{|l|}{ Actividad física } \\
\hline Sí & $30(85.7)$ & $36(66.7)$ & 0.051 \\
\hline No & $5(14.3)$ & $18(33.3)$ & \\
\hline \multicolumn{4}{|l|}{ Tipo de alimento } \\
\hline Balanceado & $24(68.6)$ & $17(31.5)$ & 0.001 \\
\hline Casero & $11(31.4)$ & $37(68.5)$ & \\
\hline
\end{tabular}

${ }^{1}$ Prueba de Chi-cuadrado, nivel de confianza del 95\%

$(\mathrm{p}=0.001)$. La edad también se encontró asociada a la presentación de obesidad $(\mathrm{p}=0.030)$, siendo más frecuente en perros entre 8 y 12 años $(51.9 \%, \mathrm{n}=28)$ (Cuadro 1).

No se encontró diferencia en obesidad según el sexo (48.1 para machos y 51.9\% para hembras. Otras variables mostraron ciertas diferencias, pero sin llegar a ser estadísticamente significativas (Cuadro 1). Así, los perros que realizaban actividad física con regularidad presentaron menores índices de obesidad (66.7\% vs. $33.3 \%)$, los perros de raza tuvieron una mayor frecuencia de obesidad $(74.1 \%, \mathrm{n}=40)$, así como aquellos que se encontraban esterilizados $(53.7 \%$, $\mathrm{n}=29$ ).

Según los resultados del análisis bivariado (Cuadro 2), consumir un alimento casero incrementa la probabilidad de desarrollar obesidad [OR 4.75; 95\%IC: $1.89-$ 11.87]. Los resultados evidenciaron a la edad como un factor asociado: los perros con edad entre 8 y 12 años tienen una probabilidad superior a 10.5 veces de desarrollar obesidad 
Cuadro 2. Modelo de regresión logística múltiple para los factores asociados a la presentación de obesidad en perros adultos atendidos en la Clínica de Animales Menores de la Universidad Nacional Mayor de San Marcos (Lima, Perú, 2017)

\begin{tabular}{|c|c|c|c|c|c|c|}
\hline Características & OR & IC 95\% & valor $\mathrm{p}$ & $\mathrm{OR}_{\mathrm{a}}$ & IC 95\% & valor $p$ \\
\hline \multicolumn{7}{|l|}{ Sexo } \\
\hline Hembra & Ref. & & & & & \\
\hline Macho & 0.88 & $0.37-2.05$ & 0.762 & 0.65 & $0.20-2.11$ & 0.477 \\
\hline \multicolumn{7}{|l|}{ Edad (años) } \\
\hline$<=1$ & Ref. & & & & & \\
\hline $2-3$ & 3.50 & $0.50-24.27$ & 0.205 & 6.83 & $0.71-65.39$ & 0.095 \\
\hline $4-7$ & 2.73 & $0.44-16.75$ & 0.279 & 6.17 & $0.68-56.09$ & 0.106 \\
\hline $8-12$ & 10.5 & $1.77-62.44$ & 0.010 & 20.13 & $2.30-176.02$ & 0.007 \\
\hline$>12$ & 5.25 & $0.69-39.48$ & 0.107 & 5.29 & $0.53-52.33$ & 0.154 \\
\hline \multicolumn{7}{|l|}{ Esterilización } \\
\hline No & Ref. & & & & & \\
\hline Sí & 1.96 & $0.82-4.68$ & 0.128 & 1.31 & $0.41-4.24$ & 0.650 \\
\hline \multicolumn{7}{|l|}{ Raza } \\
\hline Mestizo & Ref. & & & & & \\
\hline Pura & 1.31 & $0.51-3.35$ & 0.573 & 4.34 & $1.03-18.32$ & 0.045 \\
\hline \multicolumn{7}{|l|}{ Actividad física } \\
\hline No & Ref. & & & & & \\
\hline Sí & 0.33 & $0.11-1.01$ & 0.051 & 0.71 & $0.17-2.93$ & 0.641 \\
\hline \multicolumn{7}{|l|}{$\begin{array}{l}\text { Tipo de } \\
\text { alimento }\end{array}$} \\
\hline Balanceado & Ref. & & & & & \\
\hline Casero & 4.75 & $1.89-11.87$ & 0.001 & 11.5 & $2.96-44.65$ & $<0.001$ \\
\hline
\end{tabular}

ORa: OR ajustado en el modelo de regresión logística múltiple

en comparación con los perros de 1 año [OR 10.5; 95\%IC: $1.77-62.44]$. Las variables sexo [OR 0.88; 95\%IC: .0.37-20.5], esterilización [OR 1.96; 95\%IC: 0.82-4.68], actividad física [OR 0.33; 95\%IC: 0.11-1.01] y raza [OR $1.31 ; 95 \%$ IC: $0.51-3.35]$ no fueron significativas como factores asociados para la presentación de obesidad ( $p>0.05)$.

El modelo de regresión logística múltiple confirmó una asociación estadísticamente significativa para el consumo de alimento casero incrementándose a 11.5 veces la probabilidad de desarrollar obesidad en comparación a una dieta basada en alimento balanceado [OR 11.5 ; 95\%IC: 2.96-44.65]. De manera similar, se evidenció que la asociación de la edad entre 8 y 12 años aumenta hasta 20.13 veces la probabilidad de desarrollar obesidad al ser comparados con perros de 1 año [OR 20.13 ; 95\%CI:2.30176.02]. El análisis múltiple también evidenció que los perros de raza tienen 4.34 veces más probabilidad a desarrollar obesidad en 
comparación a los perros mestizos, siendo esta asociación estadísticamente significativa [OR $4.34 ; 95 \%$ CI: 1.03-18.32]. El sexo [OR 0.65 ; 95\% IC: $0.20-2.11$ ], actividad física [OR $0.71,95 \%$ IC: $0.17-2.93]$ y esterilización [OR 1.31 ; 95\% IC: 0.41-4.24] no representaron factores asociados a la presentación de obesidad en el análisis de regresión múltiple ( $\mathrm{p}>0.05$ ) (Cuadro 2).

\section{Discusión}

El estudio evidenció a las variables edad, tipo de alimentación y raza como factores asociados a la presentación de obesidad en perros. La edad entre 8 y 12 años fue el factor asociado más alto con un aumento de probabilidad de desarrollar obesidad de 20.1 veces en comparación a los perros de 1 año. Una alta frecuencia de perros con obesidad ha sido ampliamente reportada en perros de mayor edad (Robertson, 2003; McGreevy et al., 2005). El aumento de la obesidad con la edad es el resultado de la disminución de la tasa metabólica que ocurre durante el envejecimiento, donde las necesidades energéticas son inferiores (Robertson, 2003; Morrison et al., 2013). Sin embargo, los resultados del estudio también evidenciaron una disminución de esta asociación en perros mayores a 12 años en comparación con perros de 1 año [OR 5.29], lo cual podría deberse a que los perros pierden peso conforme envejecen (McGreevy et al., 2005; Bach et al., 2007; Laflamme, 2012).

Los resultados del estudio sugieren que el tipo de alimentación es un factor importante en la ocurrencia de obesidad en perros. Una alimentación basada en comida casera o mixta incrementa la posibilidad de desarrollar obesidad 11.5 veces en comparación a una dieta basada en alimento balanceado. Los alimentos comerciales para perros usualmente son de alta calidad nutricional con una fórmula basada en raciones, cubriendo los requerimientos nutricionales de los perros. A pesar de ello, existe una alta frecuencia de dueños que prefieren brindar alimento casero a sus mascotas. Muchas personas dudan del valor nutricional y calidad de ingredientes de los preparados comerciales, debido a su preocupación por la presencia de productos químicos desconocidos y el procesamiento industrial que conlleva (Oliveira et al., 2014). Sin embargo, el alimento casero puede contener altos niveles de carbohidratos y ácidos grasos saturados, generando aumento de peso y desarrollo de obesidad (Sallander et al., 2010; Heuberger y Wakshlag, 2011).

Se identificó a la raza como un factor asociado con el desarrollo de obesidad en perros. Razas como Rottweiller, Labrador, San Bernardo, Collie y Chow-Chow han sido asociadas con la presentación de obesidad (Edney y Smith, 1986). Inclusive, en perros Labrador Retriever se detectó por primera vez una mutación genética relacionada a la acumulación de tejido adiposo (Raffan et al., 2016). En el presente estudio no se discriminó por tipo de raza; sin embargo, se evidenció que los perros de raza mestiza tienen una menor probabilidad de desarrollar obesidad.

No se evidenció una asociación significativa entre la obesidad y la actividad física, similar a lo reportado por otros autores (Sallander et al., 2010; Gerstner y Liesegang, 2017). Tampoco se evidenció asociación entre la obesidad con el sexo y esterilización previa, a pesar de estar ampliamente documentado en la literatura. El sexo es un factor asociado a la obesidad en perros, siendo más frecuente en hembras (McGreevy et al., 2005; Mao et al., 2013). La esterilización también se asocia a una mayor ganancia de peso y obesidad; pues los perros esterilizados tienden a consumir mayor cantidad de alimentos y realizan un menor gasto energético diario (Jeusette et al., 2004). Sin embargo, no se evidenció una asociación estadísticamente significativa, similar a lo reportado por Markwell et al. (1990). 
Los resultados resaltan la importancia que tienen los médicos veterinarios para guiar a los dueños con una dieta balanceada para sus perros, especialmente a grupos de riesgo (edad y raza).

\section{Conclusión}

El tipo de alimento, la edad y el tipo racial fueron factores asociados con la presentación de obesidad en los perros incluidos en el estudio.

\section{Agradecimientos}

Los autores agradecen al Vicerrectorado de Investigación y Posgrado de la Universidad Nacional Mayor de San Marcos, Lima - Perú, por el financiamiento a través del Estudio de Investigación: CON CON 2014 (R.R. N 01547-R-14, Código 140801141).

\section{Literatura Citada}

1. Bach JF, Rozanski EA, Bedenice D, Chan DL, Freeman LM, Lofgren JLS, Oura TJ, Hoffman AM. 2007. Association of expiratory airway dysfunction with marked obesity in healthy adult dogs. Am J Vet Res 68: 670-675. doi: 10.2460/ajvr.68.6.670

2. Bjornvad CR, Gloor S, Johansen SS, Sandoe P, Lund TB. 2019. Neutering increases the risk of obesity in male dogs but not in bitches. A cross-sectional study of dog- and owner-related risk factors for obesity in Danish companion dogs. Prev Vet Med 170. doi: 10.1016/ j.prevetmed.2019.104730

3. Corbee RJ. 2012. Obesity in show dogs. J Anim Physiol Anim Nutr (Berl) 97: 904-910. doi: 10.1111/j.1439-0396.2012.01336.x
4. Courcier EA, Thomson RM, Mellor DJ, Yam PS. 2010. An epidemiological study of environmental factors associated with canine obesity. J Small Anim Pract 51: 362-367. doi: 10.1111/j.17485827.2010.00933.x

5. Eastland-Jones RC, German AJ, Holden SL, Biourge V, Pickavance LC. 2014. Owner misperception of canine body condition persists despite use of a body condition score chart. J Nutr Sci 3: e45. doi: 10.1017/jns. 2014.25

6. Edney AT, Smith PM. 1986. Study of obesity in dogs visiting veterinary practices in the United Kingdom. Vet Rec 118: 391-396. doi: 10.1136/ vr.118.14.391

7. German A. 2001. The growing problem of obesity in dogs and cats. J Nutr 131: 604S-615S. doi: 10.1093/JN

8. German AJ, Holden SL, WisemanOrr ML, Reid J, Nolan AM, Biourge V, Morris PJ, Scott EM. 2012. Quality of life is reduced in obese dogs but improves after successful weight loss. Vet J 192: 428-434. doi: 10.1016/ j.tvj1.2011.09.015

9. German AJ, Woods GRT, Holden SL, Brennan L, Burke C. 2018. Dangerous trends in pet obesity. Vet Rec 182: 25. doi: 10.1136/vr.k2

10. Gerstner K, Liesegang A. 2017. Survey: nutrition, body condition and activities of dogs in Switzerland. J Anim Physiol Anim Nutr (Berl) 101: 15-20. doi: 10.1111/jpn. 12615

11. Gossellin J, Wren JA, Sunderland SJ. 2007. Canine obesity - an overview. J Vet Pharmacol Ther 30: 1-10. doi: 10.1111/j.1365-2885.2007.00863.x

12. Heuberger R, Wakshlag J. 2011. The relationship of feeding patterns and obesity in dogs. J Anim Physiol Anim Nutr (Berl) 95: 98-105. doi:10.1111/ j.1439-0396.2010.01024.x 
13. Jeusette I, Detilleux J, Cuvelier C, Istasse L, Diez M. 2004. Ad libitum feeding following ovariectomy in female Beagle dogs: effect on maintenance energy requirement and on blood metabolites. J Anim Physiol Anim Nutr (Berl) 88: 117-121.

14. Kopelman PG. 2000. Obesity as a medical problem. Nature 404: 635-643. doi: $10.1038 / 35007508$

15. Laflamme D. 1997. Development and validation of a body condition score system for dogs: a clinical tool. Canine Pract 22: 10-15.

16. Laflamme DP. 2012. Nutritional care for aging cats and dogs. Vet Clin N Am Small 42: 769-791. doi: 10.1016/j.cvsm.2012.04.002

17. Lund E, Armstrong P. Jane, Kirk Claudia A., Klausner J. 2006. Prevalence and risk factors for obesity in adult dogs from private US veterinary practices. Int J Appl Res Vet Med 4:177-186.

18. Mao J, Xia Z, Chen J, Yu J. 2013. Prevalence and risk factors for canine obesity surveyed in veterinary practices in Beijing, China. Prev Vet Med 112: 438442. doi: 10.1016/j.prevetmed.2013.08.012

19. Markwell P, Van-Erk W, Parkin G, Sloth C, Shantz-Christienson T. 1990. Obesity in the dog. J Small Anim Pract 31:533-537.

20. McGreevy PD, Thomson PC, Pride C, Fawcett A, Grassi T, Jones B. 2005. Prevalence of obesity in dogs examined by Australian veterinary practices and the risk factors involved. Vet Rec 156: 695702. doi: 10.1136/vr.156.22.695

21. Morrison R, Penpraze V, Beber A, Reilly JJ, Yam PS. 2013. Associations between obesity and physical activity in dogs: a preliminary investigation. J Small Anim Pract 54: 570-574. doi: 10.1111/ jsap. 12142
22. Oliveira MCC, Brunetto MA, da Silva FL, Jeremias JT, Tortola L, Gomes MOS, Carciofi AC. 2014. Evaluation of the owner's perception in the use of homemade diets for the nutritional management of dogs. J Nutr Sci 3: 1-5. doi:10.1017/jns.2014.24

23. Raffan E, Dennis RJ, O'Donovan CJ, Becker JM, Scott RA, Smith SP, Withers DJ, et al. 2016. A deletion in the canine POMC gene is associated with weight and appetite in obesity-prone Labrador Retriever dogs. Cell Metab 23: 893-900. doi: 10.1016/j.cmet.2016.04.012

24. Reiter T, Jagoda E, Capellini TD. 2016. Dietary variation and evolution of gene copy number among dog breeds. PLoS One 11(2): e0148899. doi: 10.1371/ journal.pone.0148899

25. Robertson ID. 2003. The association of exercise, diet and other factors with owner-perceived obesity in privately owned dogs from metropolitan Perth, WA. Prev Vet Med 58: 75-83. doi: 10.1016/S0167-5877(03)00009-6

26. Rolph NC, Noble P-JM, German AJ. 2014. How often do primary care veterinarians record the overweight status of dogs? J Nutr Sci 3: e58. doi: 10.1017/jns. 2014.42

27. Sallander M, Hagberg M, Hedhammar T, Rundgren M, Lind-berg JE. 2010. Energy-intake and activity risk factors for owner-perceived obesity in a defined population of Swedish dogs. Prev Vet Med 96: 132-141. doi: 10.1016/ j.prevetmed.-2010.05.004

28. Szumilas M. 2010. Explaining odds ratios. J Can Acad Child Adolesc Psychiatry 19: 227-229.

29. Wakshlag J, Loftus J. 2014. Canine and feline obesity: a review of pathophysiology, epidemiology, and clinical management. Vet Med Res Reports 6: 49. doi: $10.2147 /$ vmrr.s40868

30. Zoran DL. 2010. Obesity in dogs and cats: a metabolic and endocrine disorder. Vet Clin N Am Small 40: 221-239. doi: 10.1016/j.cvsm.2009.10.009 